\title{
Mapping of genetic loci that modulate differential colonization by Escherichia coli 0157:H7 TUV86-2 in advanced recombinant inbred BXD mice
}

Lisa M. Russo', Nourtan F. Abdeltawab²,3, Alison D. O'Brien', Malak Kotb ${ }^{2,4}$ and Angela R. Melton-Celsa ${ }^{1 *}$

\begin{abstract}
Background: Shiga toxin (Stx)-producing E. coli (STEC) are responsible for foodborne outbreaks that can result in severe human disease. During an outbreak, differential disease outcomes are observed after infection with the same STEC strain. One question of particular interest is why some infected people resolve infection after hemorrhagic colitis whereas others progress to the hemolytic uremic syndrome (HUS). Host age and infection dose have been implicated; however, these parameters do not appear to fully account for all of the observed variation in disease severity. Therefore, we hypothesized that additional host genetic factors may play a role in progression to HUS.

Methods and Results: To mimic the genetic diversity in the human response to infection by STEC, we measured the capacity of an 0157:H7 outbreak isolate to colonize mouse strains from the advanced recombinant inbred (ARI) BXD panel. We first infected the BXD parental strains C57BL/6 J (B6) and DBA/2 J (D2) with either 86-24 (Stx2a+) or TUV86-2, an Stx2a-negative isogenic mutant. Colonization levels were determined in an intact commensal flora (ICF) infection model. We found a significant difference in colonization levels between the parental B6 and D2 strains after infection with TUV86-2 but not with 86-24. This observation suggested that a host factor that may be masked by Stx2a affects O157:H7 colonization in some genetic backgrounds. We then determined the TUV86-2 colonization levels of 24 BXD strains in the ICF model. We identified several quantitative trait loci (QTL) associated with variation in colonization by correlation analyses. We found a highly significant QTL on proximal chromosome 9 (12.5-26.7 Mb) that strongly predicts variation in colonization levels and accounts for 15-20 \% of variance. Linkage, polymorphism and co-citation analyses of the mapped region revealed 36 candidate genes within the QTL, and we identified five genes that are most likely responsible for the differential colonization.
\end{abstract}

Conclusions: The identification of the QTL on chromosome 9 supports our hypothesis that individual genetic makeup affects the level of colonization after infection with STEC O157:H7.

Keywords: QTL mapping, E. coli, O157:H7, Hemorrhagic colitis, BXD strains, Systems genetics, Genetics of susceptibility to infection, Host-pathogen interaction, Genomic biomarkers, Bioinformatics

\footnotetext{
* Correspondence: angela.melton-celsa.ctr@usuhs.edu

${ }^{1}$ Department of Microbiology and Immunology, Uniformed Services

University of the Health Sciences, Bethesda, MD, USA

Full list of author information is available at the end of the article
} 


\section{Background}

Shiga toxin (Stx)-producing E. coli (STEC) are Gramnegative enteric pathogens associated with foodborne outbreaks. E. coli O157:H7 accounts for the greatest incidence of disease due to a single STEC serotype in the in the United States. Indeed, E. coli O157:H7 is estimated to be responsible for 63,000 of the estimated 175,000 STEC cases per year [1]. Stx, the primary STEC virulence factor, is an $\mathrm{AB} 5$ toxin that inhibits protein synthesis and leads to cell death [2]. The A subunit is responsible for the catalytic activity of the molecule while the $\mathrm{B}$ pentamer binds to the host cell receptor, globotriaosylceramide (Gb3). An STEC strain may encode Stx1a and/or Stx2a, two biologically similar though antigenically distinct toxins. Some STEC strains also have the locus of enterocyte effacement (LEE) pathogenicity island [3], a large segment of 43 $\mathrm{Kb}$ DNA flanked by direct repeats, that encodes a type III secretion system and the adhesin intimin. Intimin is necessary for maximal colonization by $\mathrm{O} 157: \mathrm{H} 7$ in mouse, pig, and calf models [4-7].

Cattle and other ruminants are the natural carriers of STEC O157:H7, and contamination of the organism in meat most often occurs during beef processing [8-10]. Undercooked ground beef was responsible for the majority of STEC outbreaks initially [11, 12]; however, contaminated fresh produce and non-pasteurized beverages also spread STEC [13-17]. After ingestion of even low doses of $E$. coli $\mathrm{O} 157: \mathrm{H} 7$, the most common disease manifestation is bloody diarrhea or hemorrhagic colitis (HC) [18]. A serious sequela of $E$. coli $\mathrm{O} 157: \mathrm{H} 7$ infection, the hemolytic uremic syndrome (HUS), is defined by thrombocytopenia, hemolytic anemia, and kidney failure and occurs in 10-20\% of individuals [18]. It is not known why some individuals spontaneously clear infection while others progress to the HUS. Infectious dose and age of the patient have been implicated [19-21]; however, these factors alone do not account for the total observed disease variance $[18,22]$.

Host genetic factors are likely important determinants for O157:H7-related disease outcome. Because traditional laboratory animal models are inbred to reduce genetic heterogeneity and to minimize variably between experiments [23], a different animal model was required to investigate phenotypic variations and to reflect the complex genetic structure of the human population. The advanced recombinant inbred murine (ARI) BXD panel [24], was created by intercrossing two mouse strains, C57BL/6 J (B6) and DBA/2 J (D2). The progeny BXD strains were inbred to homozygositiy and genotyped to determine the genotypic contest of each strain and to identify the location of crossover events. The BXD panel has approximately 580,000 single nucleotide polymorphisms (SNPs) and microsatellite markers [25, 26].
In addition, the genomes of the parental parents B6 [27] and D2 $[28,29]$ were sequenced and found to differ at approximately 4.8 million SNPs. Phenotypic differences among the BXD strains are primarily related to the SNPs and 500,000 insertion-deletions [26].

Therefore, use of the BXD panel allows for a systems genetic, genome-wide analysis to facilitate identification of a quantitative trait locus (QTL) responsible for phenotypes such as variation of severity of diabetes [30], forebrain weight [31], bone density [32], or addiction response to alcohol $[33,34]$. Of particular relevance to this study, the BXD panel was used to explore genetic traits that underlie the response to infectious diseases such as influenza [35], streptococcal sepsis [36, 37], and Ebola [38] infections. A comparison of the human and murine genome reveals a high degree of similarity [39]. Therefore, it is theoretically possible to translate QTL findings from BXD mice to humans (reviewed in $[40,41]$ ). Thus, we theorized that host genetic loci that impact colonization by $\mathrm{O} 157: \mathrm{H} 7$ in the BXD panel may reflect human traits responsible for STEC disease.

In this study, we observed a statistically significant difference in colonization levels in the murine parental strains (B6 and D2) after infection with TUV86-2. That difference indicates the presence of a potential QTL involved in O157:H7 colonization that may be masked when Stx2a is expressed by the infecting $E$. coli O157:H7 strain. Analysis of colonization data from 24 BXD strains infected with TUV86-2 identified a highly significant QTL on proximal chromosome (chr) 9 between 12.5 and $26.7 \mathrm{Mb}$ that strongly predicts variation in colonization levels one day post-infection, accounting for $15-20 \%$ of variance. This QTL harbors several genes known to regulate immune responses to bacterial infections. We evaluated candidate genes within this QTL using multiple parameters that included linkage, gene ontology, variation in gene expression, co-citation networks, and biological relevance. We identified five genes of interest that may be responsible for the observed differential colonization phenotype.

\section{Methods}

\section{Ethics statement}

All animal studies were approved by the Institutional Animal Care and Use Committee of the Uniformed Services University of the Health Sciences and were conducted in strict accordance with the recommendations of the Guide for the Care and Use of Laboratory Animals [42]. Animals were housed in filter top cages with access to food and water ad libitum unless otherwise noted, in an environmentally controlled room approved by the American Association for Accreditation of Laboratory Animal Care (AAALAC). 


\section{Mice}

Female mice, approximately 5-6 weeks old were used for all experiments. BXD parental strains (B6 and D2) were purchased from The Jackson Laboratory (JAX) (Bar Harbor, Maine). We obtained some BXD strains through collaboration with investigators at the University of Cincinnati (UC) who had acquired BXD breeding pairs from the University of Tennessee Health Science Center (UTHSC) (Memphis, Tennessee) [24]. Ten BXD strains (BXD 32, 44, 49, 51, 55, 65a, 75, 86, 96, and 98) were only analyzed from UC. Additional BXD strains were from UC or JAX. Similar colonization levels between mice from UC and JAX were confirmed with four BXD strains (BXD \#: 73, 73b, 83, 87) (Additional file 1: Figure S1). Ten additional BXD strains (BXD \#: 45, 48, 61, 62, 66, 69, 70, 84, 100, and 101) were tested from both UC and JAX (Additional file 1: Figure S1), while five BXD strains (BXD \#: 60, 71, 73a, 99, 102) were only analyzed from the JAX colony. A minimum of two biological replicates were conducted for each BXD strain. We tested total of 31 strains, 29 BXD strains and ancestral parental strains B6 and D2, with 321 mice total (each BXD strain $n=6-20$; B6 and D2 $n=48$ ).

\section{E. coli 0157:H7 strains and growth conditions}

Colonization studies with the BXD parental strains (B6 and D2) were conducted with two STEC O157:H7 strains: 86-24, an Stx2a positive clinical isolate, and TUV86-2 an Stx2a-negative isogenic mutant [43-45]. BXD colonization studies were conducted only with TUV86-2. Both STEC strains are resistant to nalidixic acid (Nal) and were grown in Luria broth supplemented with $25 \mu \mathrm{g} / \mathrm{mL} \mathrm{Nal}$. To prepare the inoculum, an overnight culture $(\sim 18 \mathrm{~h})$ was pelleted by centrifugation $(5000 \times \mathrm{g})$, the supernatant removed, and the pellet resuspended 1:100 in phosphate buffered saline (PBS) supplemented with $20 \%$ sucrose. The inoculum was serially diluted and plated to determine the dose/mouse.

\section{Intact commensal flora (ICF) infection model}

Colonization levels were determined in the ICF infection model as previously reported [46]. Briefly, food and water were removed from the mice for 20 or $2 \mathrm{~h}$, respectively, prior to infection. Mice were fed a high inoculum, approximately $10^{10}$ colony forming units $(\mathrm{CFU})$ in $100 \mu \mathrm{L}$ by pipette tip. Each experiment included three mice per strain, with six to seven strains total. The parental B6 and D2 strains were included in all experiments as an internal control for colonization levels. Mice were weighed daily and colonization levels were reported as CFU per $g$ feces on day one through day four post-infection.

To determine the CFU per $\mathrm{g}$ feces, mice were placed in individual cages with no bedding for 30-40 min. After this time, mice were returned to their original cage and fecal pellets were collected, weighed, and resuspended $1: 10 \mathrm{w} / \mathrm{v}$ in PBS. The fecal slurry was further diluted 1:10 in PBS and plated on sorbitol MacConkey (SMAC) agar supplemented with $\mathrm{Nal}$ to select for the inoculating strain. The dilution that contained between 30 and 300 colonies was counted to determine CFU per $g$ feces. The limit of detection for this model is 100 CFU per g.

\section{Data analysis and QTL mapping}

We dedicated a considerable amount of time to data error checking and filtration after each experiment. Data from individual mice were flagged in the database and excluded from the final analysis if there were factors that affected colonization levels other than infection. For the final analysis we used data from 24 BXD strains and the parental mice. We performed general linear model (GLM) analysis of covariates using ordinary leastsquares analysis of variance (OLS ANOVA) to determine the relative effect and interactions of covariates on the genetic factor, represented as mouse strain. GLM analysis revealed that there were no differences associated with mouse age, source, or the seasonality of the experiment $(P>0.15)$, Additional file 2: Table S1. Mouse strain was the most significant predictor of colonization $(P<0.0001)$, followed by inoculum $(P=0.02)$. Although the differences in inoculum were statistically significant, we do not believe them to be biologically significantly, as they were within the same log for all experiments and did not result in any difference in colonization levels of the parental strains. We used the open-access web-based interval analysis on the GeneNetwork (GN) platform for complex trait analysis to identify QTLs. The primary data has been entered under trait IDs 17467, 16607, 16608, 18071, and 18072. The genome-wide interval mapping module allowed us to analyze phenotypes in the context of mouse genotypic differences and estimate the significance at each location using 5000 permutation tests [25]. We did ten sets of analyses of the log CFU means, $\log$ CFU medians or corrected coefficient of $\log \mathrm{CFU} / \mathrm{g}$ feces for the following variables: 1) colonization one day post-infection; 2) colonization two days post-infection; 3) colonization 3 days post-infection; 4) colonization 4 days post-infection; 5) difference in colonization between day four and one post-infection; 6) difference in colonization between day four and two after infection; 7) difference in colonization between day four and three after infection; 8) difference in colonization between day three and one after infection; 9) difference in colonization between day three and two after infection; and, 10) difference in colonization between day two and one after infection, for 30 traits analyzed. In addition, we determined the overall variation in median colonization across the BXD panel over time from the linear and polynomial slopes of colonization 
change per strain. Therefore 32 traits overall were mapped for QTLs.

\section{Bioinformatic analyses of mapped QTLs Haplotype analyses of significant QTL on Chr 9}

We analyzed haplotypes of the BXD strains used in this study at the significant mapped QTL on Chr 9 between 12.6 and 25.6 Mb. We downloaded the BXD genotype data set as a Microsoft excel file from http://www.genenet work.org/genotypes/BXD.geno and selected for the Chr 9 mapped QTL region and examined the genotypes of the BXD strains from that database. The different BXD strains were rank-ordered according to colonization levels on day one post-infection.

\section{Polymorphism analysis (SNP analysis)}

We did SNP analysis on genes and transcripts in the mapped QTLs associated with STEC O157:H7 differential colonization. The analysis was done on the 24 BXD strains and the parental strains B6 and D2. SNP analysis data were retrieved on March 22, 2015 from the mouse phenome database (MPD) with the SNP wizard online tool at http:// phenome.jax.org/SNP. The retrieved data originated from several databases: national center for biotechnology information (NCBI) Mouse Build 37 (known as mm9), NCBI SNP database (dbSNP) build 128 and the joint project of the European Molecular Biology Laboratory (EMBL), European Bioinformatics Institute (EBI) and the Wellcome Trust Sanger Institute, (Ensembl) build 48. SNPs among BXD strains were based on Wellcome-CTC Mouse Strain SNP Genotype Set [26].

\section{QTL heatmap}

We did correlation analyses of all traits associated with the differential colonization parameters described in the QTL analysis section above (12 traits) with QTL heatmap. QTL heatmap is a bioinformatics tool offered by the GeneNetwork (GN) platform in which a hierarchical cluster tree of the mapped set of traits is computed and distances between pairs of traits is calculated with the formula1-r, where $\mathrm{r}$ is the Pearson product-moment correlation.

\section{Candidate gene co-citation analysis}

We did co-citation analyses using open-source Chilibot platform (www.chilibot.net), a literature search engine that identifies all relevant relationships among search terms [47]. We cross-referenced the 36 candidate polymorphic genes using key words associated with STEC O157:H7 colonization to identify genes most likely responsible for the QTL on Chr 9. The initial key words were: STEC; colonization; normal flora; colonization resistance; epithelial cell; tight junction; cell polarity; adhesion; mucus; mucin; nucleolin. The key words for the final interaction diagram, chosen because of the high degree of interconnectivity to the top genes and for clarity of the figure were: STEC; colon; mucus; colonization.

\section{Statistical analysis}

All primary calculations and sorting operations were done with features and functions of Microsoft Excel. Statistical analyses were executed with Data Desk (version 6.3) software (Data Description, Inc., Ithaca, NY; www.datadesk.com) and included correlation, regression, and general linear model (GLM) analyses by ordinary least squares analysis of variance (OLS ANOVA). Mouse age, weight, inoculum, and log CFU/g feces for each day post-infection were analyzed to determine the possible effect of those variables on the final results.

\section{Data deposition}

Our data sets were stored as part of the BXD published phenotypes on Gene Network Platform and can be found at (www.genenetwork.org) under BXD published phenotypes record trait IDs 17467, 16607, 16608, 18071 , and 18072.

\section{Results}

Infection with TUV86-2, but not 86-24, results in significant colonization differences between the parental murine strains

We used the ICF infection model to determine the colonization levels of two isogenic STEC O157:H7 strains in the BXD parental mice. After infection with $86-24$, an Stx2a-positive strain, both B6 and D2 mice had an average colonization level of $10^{6}-10^{7} \mathrm{CFU} / \mathrm{g}$ feces on day one postinfection (Fig. 1a). The colonization levels did not vary significantly between B6 and D2 over the course of the experiment, and both murine strains maintained colonization through day 4. We next determined colonization levels from B6 and D2 mice infected with TUV86-2, an Stx2a-negative isogenic mutant. The initial TUV86-2 colonization levels were similar to those after infection with 86-24: the geometric mean colonization levels of the parental B6 and D2 murine strains was $10^{6}$ or $10^{8} \mathrm{CFU} / \mathrm{g}$ feces, respectively (Fig. 1b). However, a significant difference in colonization levels developed by day three post-infection, such that D2 mice maintained colonization while $\mathrm{B} 6$ mice began to resolve the infection (Fig. 1b). The significant difference in colonization was maintained on day 4.

\section{Colonization differences between parental and BXD strains infected with TUV86-2}

Since there was a significant difference in the colonization levels of the parental mice after infection with TUV86-2, we decided to infect the BXD mice only with TUV86-2. All of the BXD strains tested became colonized with TUV86-2 after oral inoculation with the organism. Although the 


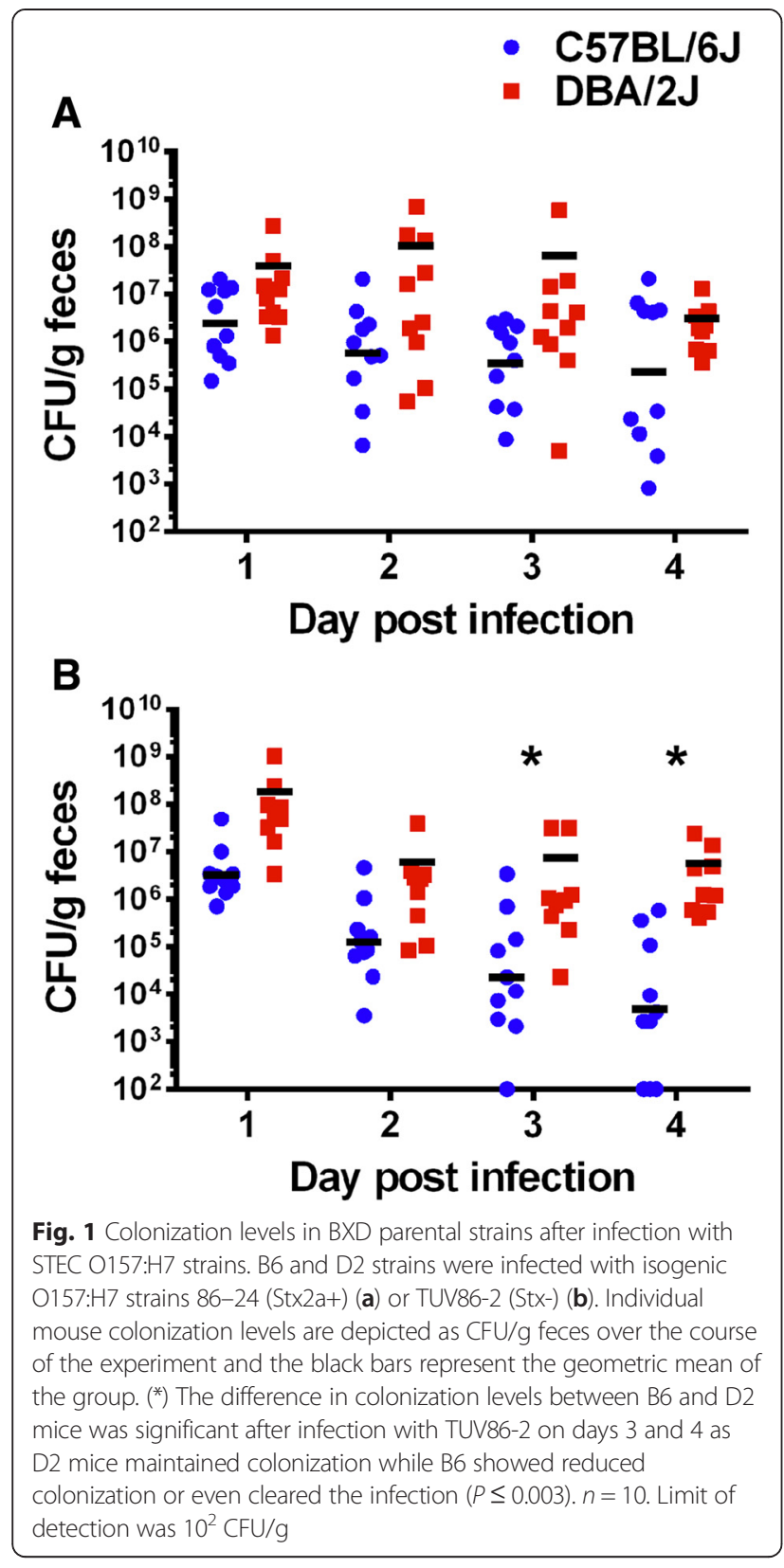

mean colonization levels of the parental murine strains one day post-infection were $1.38 \times 10^{6}$ or $1.15 \times 10^{7} \mathrm{CFU} / \mathrm{g}$ feces, respectively, for B6 and D2 mice, the mean colonization levels from the different BXD strains one day post-infection ranged from $10^{4}$ to $10^{7} \mathrm{CFU} / g$ feces (Fig. 2). Additionally, individual BXD strains exhibited different patterns of colonization over the course of the infection. A few strains maintained colonization (BXD 99 and 102), others steadily lost colonization (BXD 51, 75, 96, 97), and some others showed variable colonization over the experiment (BXD 60, 62, 71, 87, 100) (Fig. 2). These data demonstrate variable susceptibility to $\mathrm{O} 157: \mathrm{H} 7$ colonization within the BXD panel and suggest that colonization levels might be used to identify host genetic factors associated with the capacity of STEC to establish infection.

QTL identified on proximal Chr 9 associated with TUV86-2 colonization in BXD mice

We performed genome-wide scans with bioinformatics tools provided by GeneNetwork (www.genenetwork.org) to assess the observed colonization levels against the known genotypes of the BXD strains. We analyzed TUV86-2 colonization levels in the BXD strains by the 32 parameters listed in the methods. We identified a significant QTL on proximal Chr 9 when we mapped the $\log$ of the colonization means from day 1 (Fig. 3a), with a likelihood ratio statistic (LRS) of 20.19 [limit of detection $(\mathrm{LOD})=4.4$ and $P<0.05]$ and a total interval width of $14 \mathrm{Mb}$ (12.5-26.7 Mb) (Fig. 3b). We next did linkage analysis of the QTL on proximal Chr 9 and found that the QTL was linked with three genetic markers, gnf09.010.169, rs13480073, and mCV24962297 (13.23-15.69 Mb), with a peak LRS at $15.69 \mathrm{Mb}$ associated with genetic marker gnf09.010.169. When we mapped colonization levels on day 2 or 4 post-infection, we found a suggestive QTL that overlapped the Chr 9 QTL for day one colonization at interval 13-26 Mb (Table 1).

We also identified suggestive QTLs that overlapped on Chr 14 for colonization levels on days one or two postinfection with a peak LRS of 13.84 and 12.09, respectively (Table 1). We further identified multiple suggestive QTLs for the following traits: difference in colonization between two independent days post-infection [such as colonization day two minus colonization day 1 (QTL on Chr 13)], and the linear (Chr 17) and polynomial slopes of colonization change (Chr X) (Table 1).

We identified the haplotypes of the BXD strains at the significant QTL on Chr 9 between 12.6 and $25.6 \mathrm{Mb}$ and rank-ordered BXD strains according to colonization levels from low to high (Fig. 4). Strain distribution patterns (SDP) of the BXD strains revealed that high colonization levels on day one post-infection were associated with the $\mathrm{B}$ allele (blue) inherited from the parent B6. Low colonization levels in the BXD panel were associated with D alleles (red) inherited from the D2 parent. Taken together the SDP of the haplotypes suggests that overall the B allele exhibited dominance for high colonization. In addition, we performed QTL heatmap analysis that entailed correlation analyses for 12 traits associated with differential colonization (Additional file 3: Figure S2). The phylogenetic tree at the top of the QTL heatmap indicates how closely related the independent traits are to each other. We observed that the significant mapped QTL on Chr 9 was associated with B allele dominance (dark blue) in accordance with haplotype analyses. Other mapped QTLs on Chrs 1 and 5 had similar B allele dominance. In 


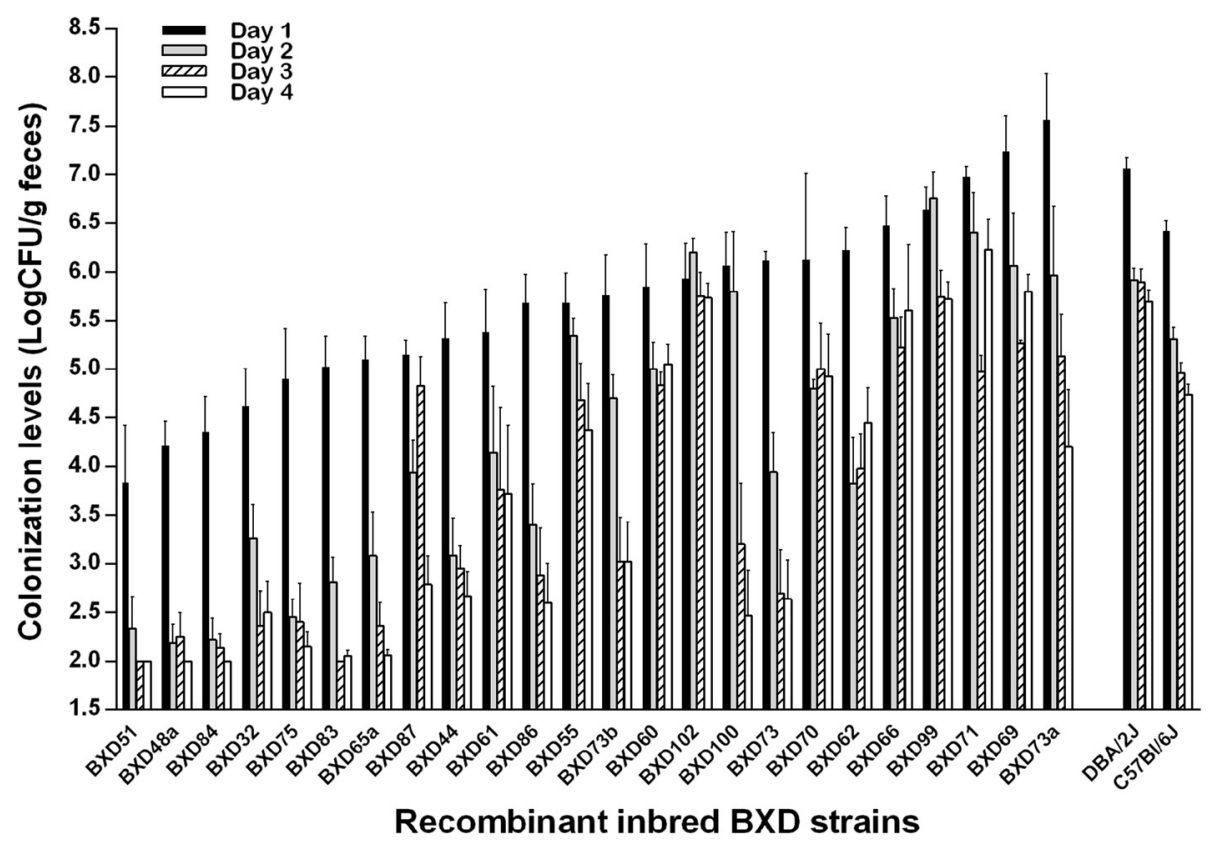

Fig. 2 BXD colonization levels after infection with TUV86-2. The TUV86-2 colonization levels for the BXD and parental murine strains over the course of the infection. Individual murine strains (sorted based on day one colonization from lowest to highest) are listed along the $x$-axis and daily colonization levels are depicted as the $\log$ CFU/g feces. Parental $n=31 ; \mathrm{BXD} n=3-9$ per strain; 182 mice total. Limit of detection was $10^{2}$ CFU/g

contrast, QTLs on Chrs 14, 15 18, and 19 had D allele dominance (Additional file 3: Figure S2).

\section{Candidate genes analyses}

We did gene enrichment analyses of the significant QTL mapped on Chr 9 with multiple parameters that included linkage, gene ontology, variation in gene expression, polymorphism, co-citation networks, and biological relevance. Polymorphism (SNP) analysis identified 36 candidate genes that might modulate differential colonization associated with the identified QTL on proximal Chr 9. SNPs were identified by the Mouse Phenome Database (http:// phenome.jax.org/). We focused on nonsynonymous SNPs, even those located within exons since those SNPs may influence translation. We found 38 SNPs of interest (Fig. 5) and with the ToppGene suite (https://toppgene.cchmc.org/) we identified 36 candidate genes (Table 2). Finally, we did co-citation networks and biological function analyses for candidate genes and key words (listed in methods). Through those analyses, we identified five genes that are most likely to modulate differential colonization. These are Pannexin 1(Panx1); BMP binding endothelial regulator (Bmper); DNA methyltransferase 1 (Dnmt1); phosphodiesterase 4A (Pde4a); and acyl-CoA dehydrogenase family, member 8 (Acad8). A visual representation of the relationship between the final key words (STEC; colonization, mucus, colon) and the five genes of interest is shown in Fig. 6.

\section{Discussion}

The major finding from this study was the identification of a significant QTL on proximal Chr 9 associated with TUV86-2 colonization levels in BXD mice one day postinfection. The identification of this QTL supported our hypothesis that host genetics affect STEC O157:H7 colonization levels in mice. Since establishment of infection is critical for comparison of colonization levels across multiple experiments, we included the BXD parental strains in every experiment as an internal control. Because the B6 and D2 day one colonization levels were consistently within the expected range $\left(10^{6}-10^{8}\right)$, we are confident that the variation in BXD colonization levels is due to genotypic differences among the strains. The variation in colonization levels across BXD strains is consistent with the mosaic genotypes of the panel. We predict that such genetic predispositions to low or high initial colonization levels could influence the severity of disease from an STEC infection. The mosaic-like genetic complexity of the ARI BXD panel provided the diversity required to map the QTL and would allow us to predict if an individual animal would be susceptible or relatively resistant to O157:H7 colonization.

We initially determined the colonization profiles of the parental murine strains after infection with both 86-24, an Stx2a-positive strain, and TUV86-2, an Stx2a-negative isogenic strain. However, since there was no difference in colonization levels of the parental strains after 86-24 infection, we decided to only infect the BXD strains with TUV86-2 to have the greatest chance of identifying a QTL 


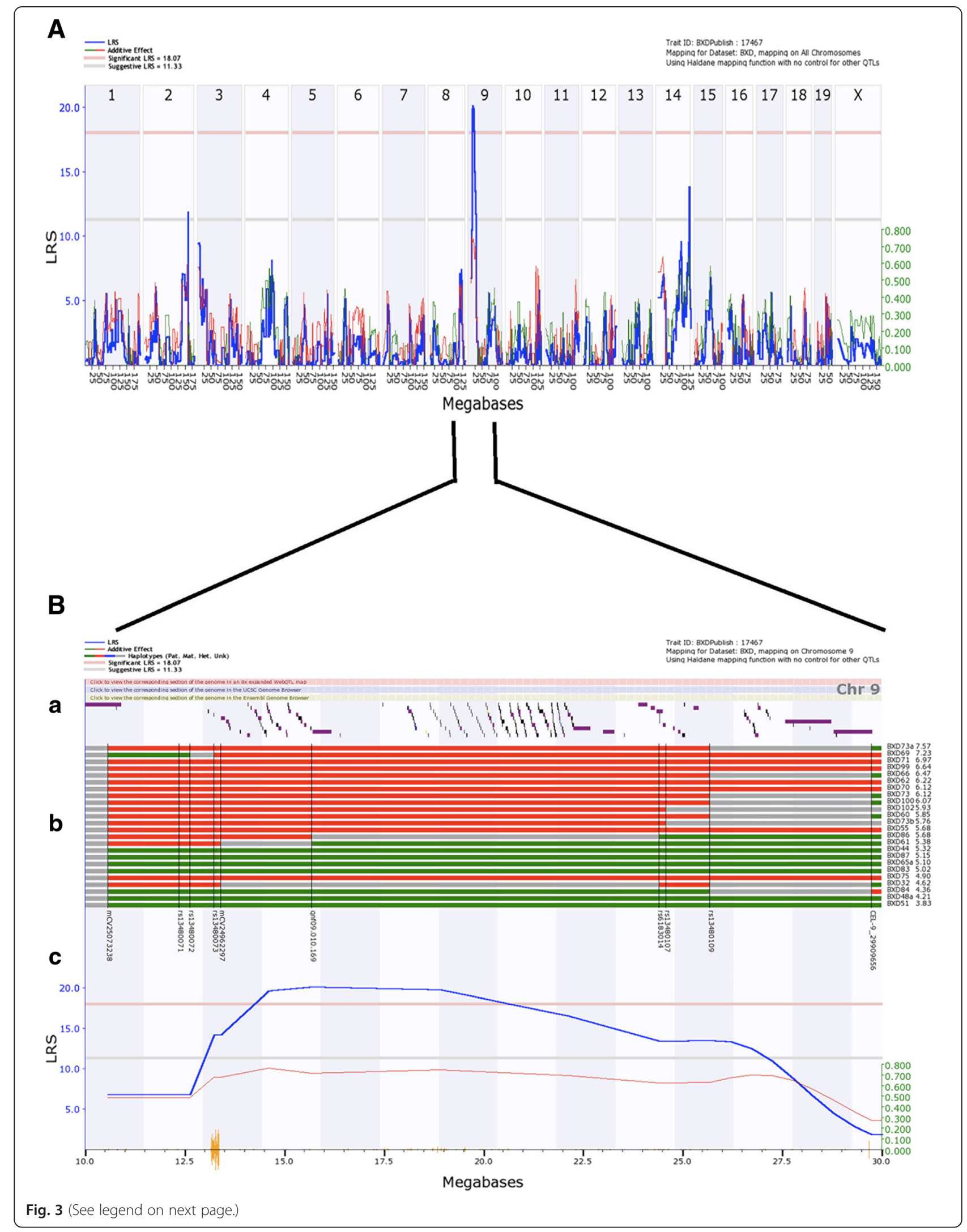


(See figure on previous page.)

Fig. 3 Genome wide scan for TUV86-2 susceptibility revealed a significant QTL on chromosome 9. a) A mouse chromosome interval map based on TUV86-2 colonization levels on day one. The $x$-axis depicts the physical map of the entire murine genome. The left $y$-axis illustrates the LRS (blue line) as an expression of strength of the association between colonization and genotypic markers. The grey line at $y=11.25$ indicates the suggestive threshold, while the pink line at $y=17.88$ shows the significant threshold. A significant QTL was identified on proximal Chr 9. b) Expanded physical map of Chr 9 for the region of the QTL. (a) Colored blocks represent the location of individual genes along the Chr. with links to corresponding section of the genome in the UCSC Genome Browser, the Ensembl Genome Browser, and expanded WebQTL map. (b) Haplotype map of BXD strains (listed on the left side with the log geometric mean colonization on day one listed next to the strain name), where green denotes D2 (paternal), red designates B6 (maternal), blue shows heterozygous and grey indicates an unknown genotype. Genetic markers associated with the mapped QTLs are shown: proximal mCV25073238, rs13480072, rs13480071, rs13480073, mCV24962297, gnf09.010.169 (associated with highest LRS), rs6183014, rs13480107, rs13480109 and CEL-9_29909656. (c) Expanded view of the QTL (blue line) overlaid on the SNP seismograph track, where each orange hash mark indicates a unique SNP. The right $y$-axis represents the additive allele effect and the red line signifies that the B6 allele is associated with increased colonization levels

associated with colonization. The reason that we did not observe a difference in colonization levels after infection by $86-24$ is most likely due to the fact that Stx2a enhances STEC colonization levels in a traditional mouse model [45]. The decision to proceed with the toxin-negative strain enabled us to identify host genetic markers that may be associated with O157:H7 colonization. Future studies could determine whether the toxin positive strain would overcome the low colonization phenotype associated with the "D" allele at the Chr 9 QTL.

Suggestive QTLs linked to colonization levels on days two and four post-infection were also mapped to

Table 1 Summary of TUV86-2 colonization QTLs in ARI BXD mice

\begin{tabular}{|c|c|c|c|c|}
\hline Name of mapped trait (Phenotype) & $\begin{array}{l}\text { Chr } \\
\left(\mathrm{mm} 9^{b}\right)\end{array}$ & $\begin{array}{l}\text { Peak } \\
\text { LRS }\end{array}$ & $\begin{array}{l}\text { Genetic marker(s) associated with } \\
\text { peak locus }\end{array}$ & $\begin{array}{l}\text { Location of genetic } \\
\text { markers (Mb) }\end{array}$ \\
\hline \multirow[t]{2}{*}{ Colonization one day after infection } & 9 & $20.19^{c}$ & $\begin{array}{l}\text { gnf09.010.169, rs13480073, and } \\
\text { mCV24962297 }\end{array}$ & $13.23-15.69$ \\
\hline & 14 & 13.84 & $\begin{array}{l}\text { rs13482392, gnf14.114.290, } \\
\text { rs13482396 }\end{array}$ & $118.97-119.71$ \\
\hline \multirow[t]{2}{*}{ Colonization two days after infection } & 9 & 16.12 & gnf09.010.169 & $13.23-25.69$ \\
\hline & 14 & 12.09 & $\begin{array}{l}\text { rs13482396, gnf14.085.610 and } \\
\text { rs3707842 }\end{array}$ & $91.32-120.03$ \\
\hline Colonization four days after infection & 9 & 12.29 & $\begin{array}{l}\text { gnf09.010.169, rs13480073, } \\
\text { mCV24962297 }\end{array}$ & $13.23-25.69$ \\
\hline Difference in colonization between day two and one after infection & 13 & 12.05 & rs6209128 and rs3023086 & $52.86-53.52$ \\
\hline \multirow[t]{3}{*}{ Difference in colonization between day three and one after infection } & 7 & 13.36 & rs6206014 & 47.86 \\
\hline & 18 & 11.98 & rs3718618 and rs3669949 & $69.37-69.9$ \\
\hline & 19 & 12.99 & $\begin{array}{l}\text { rs13483513, gnf19.005.316, } \\
\text { rs4232041 and rs4232042 }\end{array}$ & $3.41-10.15$ \\
\hline \multirow[t]{2}{*}{ Difference in colonization between day four and one after infection } & 5 & 12.86 & rs13478413 and rs3688859 & $98.23-100.05$ \\
\hline & 17 & 11.6 & rs13483110 & 76.56 \\
\hline \multirow[t]{3}{*}{ Difference in colonization between day three and two after infection } & 4 & 15.52 & rs3719891-rs6358921 & $140.88-150.45$ \\
\hline & 10 & 12.04 & rs13459120, rs13480580 & $35.75-36.17$ \\
\hline & 15 & 12.02 & rs4230714-rs3701428 & $44.2-55.00$ \\
\hline \multirow[t]{3}{*}{ Difference in colonization between day four and two after infection } & 1 & 11.66 & rs13475818 and UT_1_38.719268 & 38.07 \\
\hline & 15 & 11.28 & rs3717268 and rs13482709 & $90.7-91.5$ \\
\hline & $x$ & 16.64 & $\begin{array}{l}\text { rs13483746, rs13483748 and } \\
\text { rs13483736 }\end{array}$ & $44.68-48.08$ \\
\hline Difference in colonization between day four and three after infection & 12 & 11.17 & rs13481566-rs13481579 & $85.87-89.93$ \\
\hline $\begin{array}{l}\text { Overall strain specific variation in pattern of colonization across BXD } \\
\text { strains (Linear slopes of medians) }\end{array}$ & 17 & 12.47 & rs13483110 & 76.5 \\
\hline $\begin{array}{l}\text { Overall strain specific variation in pattern of colonization across BXD } \\
\text { strains (Polynomial slopes of medians) }\end{array}$ & $x$ & 12.17 & $\begin{array}{l}\text { rs13483770, gnfX.044.260, } \\
\text { rs13483785, rs13483786 }\end{array}$ & $56.48-61.86$ \\
\hline
\end{tabular}




\begin{tabular}{|c|c|c|c|c|c|c|c|c|c|c|c|c|c|c|c|c|c|c|c|c|c|c|c|c|c|}
\hline $\begin{array}{c}\text { Locus } \\
\text { (genetic marker) }\end{array}$ & $\begin{array}{l}\text { Location } \\
\text { (Mb) }\end{array}$ & 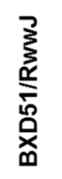 & 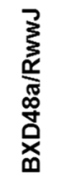 & 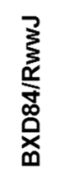 & 疋 & 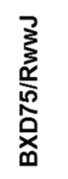 & 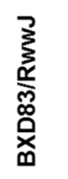 & 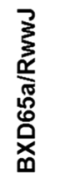 & 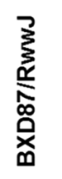 & 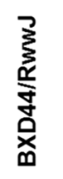 & 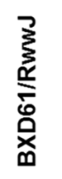 & 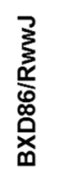 & 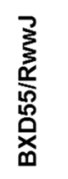 & 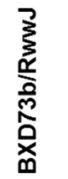 & 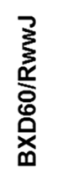 & 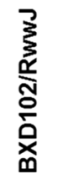 & 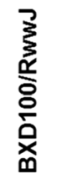 & 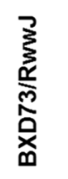 & 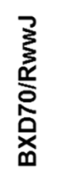 & 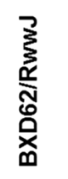 & 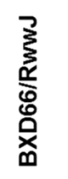 & 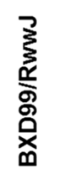 & 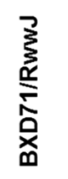 & 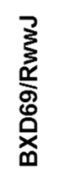 & 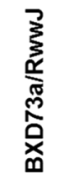 \\
\hline rs 13480072 & 12.6418 & D & $\mathrm{D}$ & D & B & B & D & $\mathrm{D}$ & D & D & B & B & B & B & B & $\mathrm{B}$ & B & B & B & B & B & B & B & D & B \\
\hline rs13480073 & 13.2338 & D & D & D & B & B & D & D & D & D & B & B & B & B & B & B & B & B & B & B & B & B & B & B & B \\
\hline mCV24962297 & 13.4047 & D & D & D & B & B & D & D & D & D & B & B & B & B & B & B & B & B & B & B & B & B & B & B & B \\
\hline gnf09.010.169 & 15.6937 & $\mathrm{D}$ & $\bar{D}$ & $\bar{D}$ & $\bar{D}$ & B & D & D & D & D & $\bar{D}$ & $B$ & B & B & B & B & B & B & B & B & B & B & B & B & B \\
\hline rs6183014 & 24.4207 & D & D & D & B & B & D & D & D & D & D & D & B & B & B & B & B & B & B & B & B & B & B & B & B \\
\hline rs 13480107 & 24.5939 & D & D & D & B & B & D & D & D & D & D & D & B & B & B & B & B & B & B & B & B & B & B & B & B \\
\hline rs 13480109 & 25.6976 & D & D & D & B & $\bar{B}$ & $\bar{D}$ & D & $\bar{D}$ & $\bar{D}$ & D & D & B & U & B & $\bar{U}$ & $\bar{B}$ & B & B & B & B & B & B & B & B \\
\hline \multicolumn{2}{|c|}{ Mice $(n)$} & 3 & 8 & 5 & 5 & 4 & 9 & 5 & 8 & 6 & 5 & 5 & 5 & 5 & 6 & 6 & 3 & 5 & 4 & 4 & 4 & 5 & 4 & 3 & 3 \\
\hline \multicolumn{2}{|c|}{$\begin{array}{l}\text { Log mean colonization, } \\
\text { day } 1 \text { (CFU/g feces) }\end{array}$} & 3.83 & 4.21 & 4.36 & 4.62 & 4.90 & 5.02 & 5.10 & 5.15 & 5.32 & 5.38 & 5.68 & 5.68 & 5.76 & 5.85 & 5.93 & 6.07 & 6.12 & 6.13 & 6.23 & 6.48 & 6.64 & 6.98 & 7.23 & 7.57 \\
\hline
\end{tabular}

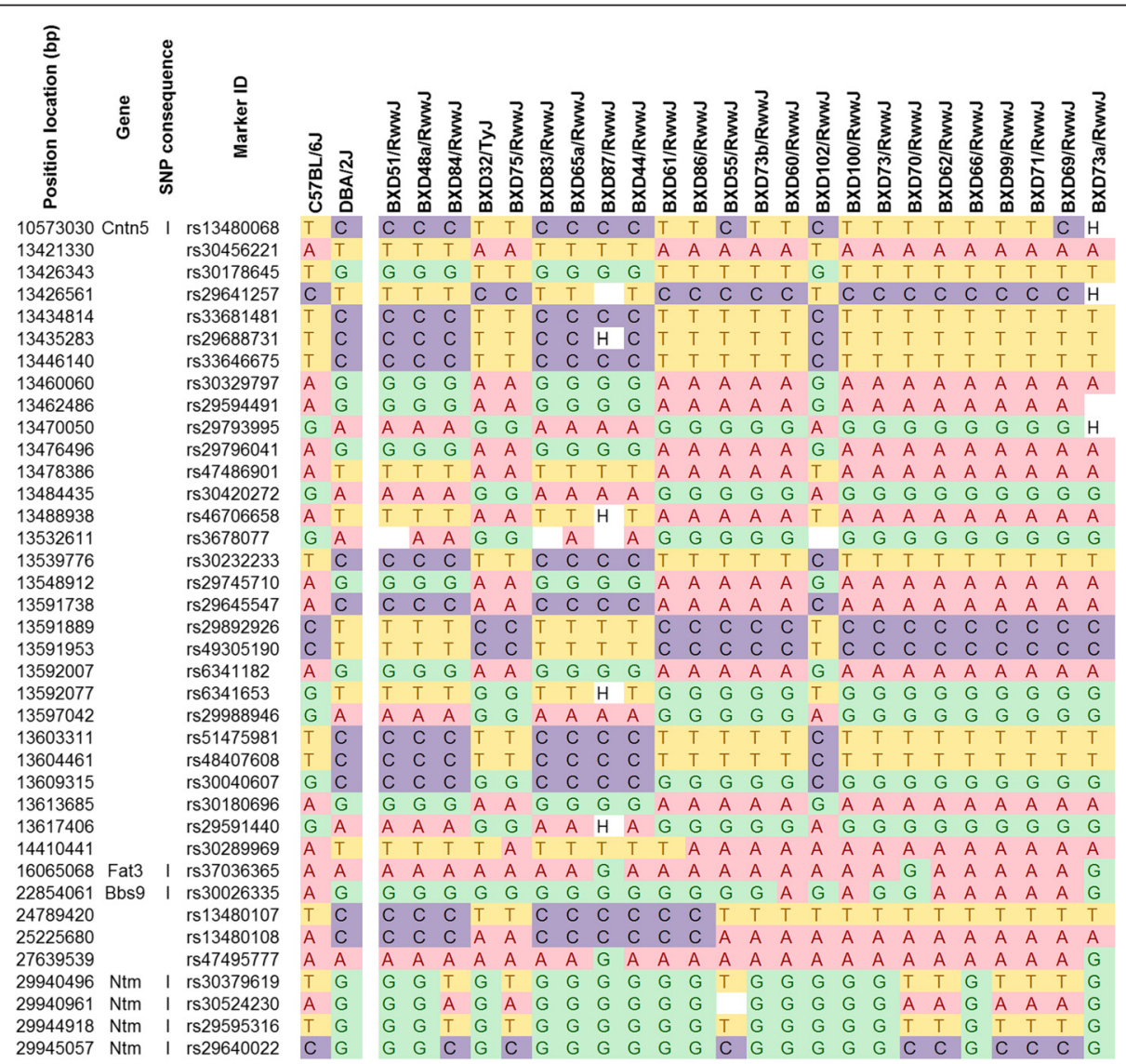

Fig. 5 Selected SNPs from the Chr 9 QTL. The SNPs of the parental and BXD strains are identified at 38 nonsynonymous loci of interest. The parental strains are shown first and the BXD strains are listed in order from lowest to highest colonization levels one day post-infection. Low colonization was associated with the D2 haplotype and high colonization was associated with the B6 haplotype. SNP consequence "I": SNP occurs within an intron. Haplotype "H": heterozygous; blank white box: unknown 
Table 2 Candidate genes on Chr 9

\begin{tabular}{|c|c|c|c|c|}
\hline Gene symbol & Name or description & Chr location (GRCm38) & Size & Identifiers \\
\hline Cntn5 & Contactin 5 & 9:9660891-10904775 - & 1243884 & MGI:3042287 \\
\hline Maml2 & Mastermind like 2 (Drosophila) & $9: 13619990-13709388+$ & 89398 & MGI:2389460 \\
\hline Sesn3 & sestrin 3 & $9: 14276301-14326138+$ & 49837 & MGl:1922997 \\
\hline Amotl1 & Angiomotin-like 1 & 9:14541966-14615483 - & 73517 & MGl:1922973 \\
\hline Mre11a & Meiotic recombination 11 homolog A (S. cerevisiae) & 9:14784654-14837123 + & 52469 & MGI:1100512 \\
\hline Panx1 & Pannexin 1 & 9:15005161-15045478 - & 40317 & MGl:1860055 \\
\hline 4931406C07Rik & RIKEN cDNA $4931406 C 07$ gene & 9:15283337-15306448 - & 23111 & MGI:1918234 \\
\hline Taf1d & $\begin{array}{l}\text { TATA box binding protein (Tbp)-associated } \\
\text { factor, RNA polymerase I, D }\end{array}$ & 9:15306214-15312104 + & 5890 & MGI:1922566 \\
\hline Ccdc67 & coiled-coil domain containing 67 & 9:15559864-15627914 - & 68050 & MGI:2443026 \\
\hline 4930540M03Rik & RIKEN cDNA 4930540 M03 gene & $9: 15619857-15641220+$ & 21363 & MGl:1925275 \\
\hline Fat3 & FAT tumor suppressor homolog 3 (Drosophila) & 9:15910205-16378231 - & 468026 & MGI:2444314 \\
\hline Naalad2 & N-acetylated alpha-linked acidic dipeptidase 2 & 9:18321951-18402995 - & 81044 & MGl:1919810 \\
\hline Olfr39 & Olfactory receptor 39 & $9: 20282351-20286648+$ & 4297 & MGl:1313142 \\
\hline Olfm2 & Olfactomedin 2 & 9:20667986-20728219 - & 60233 & MGl:3045350 \\
\hline Col5a3 & Collagen, type V, alpha 3 & 9:20770050-20815067 - & 45017 & MGI:1858212 \\
\hline Dnmt1 & DNA methyltransferase (cytosine-5) 1 & 9:20907206-20959888 - & 52682 & MGI:94912 \\
\hline Pde4a & Phosphodiesterase 4A, cAMP specific & 9:21165714-21213248 + & 47534 & MGI:99558 \\
\hline IIf3 & Interleukin enhancer binding factor 3 & 9:21368019-21405361 + & 37342 & MGI:1339973 \\
\hline Carm1 & Coactivator-associated arginine methyltransferase 1 & $9: 21546894-21589487+$ & 42593 & MGl:1913208 \\
\hline Dock6 & Dedicator of cytokinesis 6 & 9:21800184-21852635 - & 52451 & MGl:1914789 \\
\hline Gm6484 & Predicted gene 6484 & 9:21835510-21837346 + & 1836 & MGl:3643534 \\
\hline Zfp599 & Zinc finger protein 599 & 9:22247430-22259895 - & 12465 & MGI:2679006 \\
\hline Zfp810 & Zinc finger protein 810 & $9: 22276748-22307638-$ & 30890 & MGI:2384563 \\
\hline Bbs9 & Bardet-Biedl syndrome 9 (human) & 9:22475715-22888280 + & 412565 & MGI:2442833 \\
\hline Bmper & BMP-binding endothelial regulator & 9:23223076-23485202 + & 262126 & MGl:1920480 \\
\hline Npsr1 & Neuropeptide S receptor 1 & $9: 24097996-24316398+$ & 218402 & MGI:2441738 \\
\hline Dpy19|1 & dpy-19-like 1 (C. elegans) & $9: 24411776-24503140-$ & 91364 & MGl:1915685 \\
\hline Dpy 1912 & dpy-19-like 2 (C. elegans) & 9:24557048-24696293 - & 139245 & MGI:2444662 \\
\hline Tbx20 & T-box 20 & 9:24720812-24774303 - & 53491 & MGl:1888496 \\
\hline Sept7 & Septin 7 & $9: 25252439-25308571+$ & 56132 & MGl:1335094 \\
\hline Eepd1 & $\begin{array}{l}\text { Endonuclease/exonuclease/phosphatase family } \\
\text { domain containing } 1\end{array}$ & 9:25481547-25604110 + & 122563 & MGl:1914734 \\
\hline Gm1110 & Predicted gene 1110 & 9:26879567-26923081 - & 43514 & MGI:2685956 \\
\hline Acad8 & Acyl-Coenzyme A dehydrogenase family, member 8 & 9:26974135-26999566 - & 25431 & MGl:1914198 \\
\hline Ncapd3 & Non-SMC condensin II complex, subunit D3 & 9:27030175-27095311 + & 65136 & MGI:2142989 \\
\hline Opcml & Opioid binding protein/cell adhesion molecule-like & 9:27790775-28925410 + & 1134635 & MGI:97397 \\
\hline $\mathrm{Ntm}$ & Neurotrimin & 9:28994750-29963141 - & 968391 & MGI:2446259 \\
\hline
\end{tabular}

Chr 9 in the same region as the significant day one colonization QTL. In addition, an LRS that approached a suggestive QTL in the same Chr 9 region was mapped for colonization levels on day three post infection (data not shown), and the QTL heatmap for day three colonization also indicates $\mathrm{B}$ haplotype (blue color) dominance (Additional file 1: Figure S1). It is possible that the addition of more strains to the panel would strengthen the suggestive QTLs for colonization on days 3 and 4 post infection, time points for which days the parental mice also showed a difference in colonization. We believe that the overlap of multiple QTLs in one location bolsters the likelihood that this region of $\mathrm{Chr}$ 9 is tightly linked to colonization capacity. However, it 


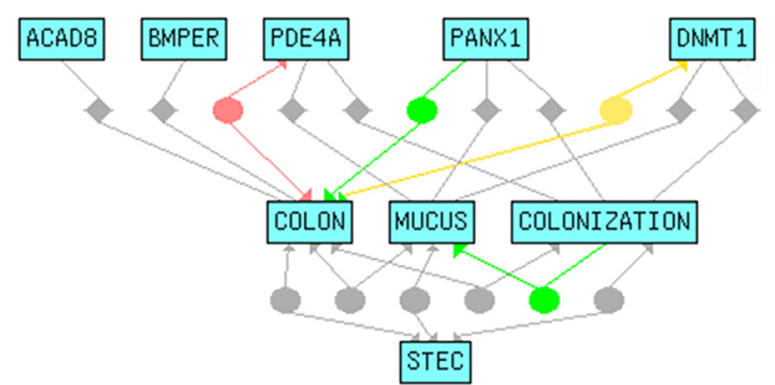

Fig. 6 Gene interaction analysis of the five genes predicted to be responsible for the Chr 9 QTL. The final 4 key words (STEC; colonization; colon; mucus) and the interactions between and among the five genes predicted to be important for the QTL: Acad8; Bmper; Pde4a; Panx1; Dnmt1. Circles indicate an interactive relationship while diamonds indicate that a co-occurrence was found. A green line indicates a stimulative relationship; a red line signifies an inhibitory relationship; a yellow line shows both stimulative and inhibitory relationships; and a grey line denotes a neutral relationship. The Chilibot search was conducted on April 9, 2015. There were nine search terms and 55 searches were conducted. A total of 2364 PubMed records were searched with 239 (10.1\%) records processed and 20 links were found

is possible that different host factors are responsible for variations in colonization on day one compared to colonization persistence as measured on days 2 and 4 . The additional suggestive QTLs identified in Table 1 linked to multiple traits illustrate the complexity of host genetic factors that respond to the presence of a bacterial pathogen. The QTLs associated with the differences in colonization between the days after infection are likely related to colonization persistence. That multiple QTLs on different Chrs are implicated in the persistence phenotype suggests that genes located across QTLs may be connected through similar pathways. This is especially evident by the three suggestive QTLs linked to the difference in colonization between day 3 and day 1 on Chrs 7, 18, 19.

Haplotype analysis in the region of Chr 9 that contains the significant QTL revealed that the D allele is associated with low colonization levels of BXD strains, a finding that contrasts with the colonization data from the parental strains in which the D allele was associated with high, sustained colonization. When there is such a reversal of SDP the QTL is referred to as a "cryptic" QTL, and is likely a reflection of the fact that the BXD panel was derived from the F2 progeny of the initial D2 x B6 cross [48]. It may be that the D2 have other factors that allow high colonization levels that mask the cryptic QTL on Chr 9. Future studies to test that latter hypothesis would determine the colonization levels of additional BXD strains that are "D" at the Chr 9 QTL to try to map a host factor(s) that allows high colonization in DBA but not the progeny.

We focused our QTL analysis on the significant QTL on proximal Chr 9. The QTL is located in a chromosomal region with limited SNPs. We chose the 36 candidate genes based on the highest number of polymorphisms, because such polymorphisms are more likely to be responsible for the QTL. We used Chilibot to compare scientific literature that included one or more of the 36 genes and key words associated with STEC colonization in the colon. Fig. 6 represents the final interconnectivity plot of the five genes most likely to be linked to the QTL: Acad8; Bmper; Pdea; Panx1; Dnmt1. All five of the genes selected are expressed in the colon of humans and mice. Acad8 is a member of the acyl-Coenzyme A dehydrogenase family and necessary for butyrate oxidation [49]. Butyrate, a short chain fatty acid, is an important source of energy for colonic enterocytes $[50,51]$ and helps to maintain intestinal epithelial cell physiology [52]. Defects in butyrate oxidation are linked to mucosal inflammation and ulcerative colitis [53]. Additionally, butyrate increases STEC adherence to $\mathrm{CaCo} 2$ cells [54] and increases the concentration of the Stx receptor, Gb3, on intestinal epithelial cells [55]. Polymorphisms of Acad8 may drive the QTL on Chr 9 by affecting the health of colonic enterocytes, which in turn promote or inhibit colonization.

Bmper, Pde4a, Panx1, and Dnmt1 are four genes that modulate inflammation of colonic enterocytes. Bmper is a BMP-binding endothelial regulator [49]. It limits endothelial inflammation by inhibiting expression of intercellular adhesion molecule 1 (ICAM1) $[56,57]$ and by regulating leukocyte extravasation and adhesion [58]. Pde4a is a cAMP-specific phosphodiesterase 4A that participates in multiple signal transduction pathways, such as platelet aggregation and immune cell activation [49]. Use of Pde4a-specific inhibitors has anti-inflammatory effects, such as reduced neutrophil adhesion [59] and inhibition of cellular trafficking and microvascular leakage [60]. Additionally, Pde4 inhibitors have been proposed to prevent STEC-mediated brain damage [61]. Panx1 is part of the innexin family, and as such a structural component of gap junctions [49] Panx1 is responsible for the release of ATP to the extracellular space, which can initiate cellular migration and inflammation [62]. Additionally, ATP release can modulate mucus secretion [63] which may impact colonization. Dnmt1 is a DNA methyltransferase responsible for the establishment and regulation of tissue specific methylated cytosine residues [49]. Since Dnmt1 activity affects global methylation patterns, variation in expression can change epithelial cell morphology [64]. Finally, Dnmt1 levels are elevated in response to UPEC infection [65]. Variation of inflammation levels could affect initial colonization, while the effect on leukocyte transit could impact colonization persistence. Further studies are needed to confirm or refute the actual involvement of one or more of the identified genes. The long term goal of this project is to correlate the genes identified within the 
murine QTL(s) with human host factor(s) responsible for the variable disease states observed during STEC O157:H7 outbreaks.

\section{Conclusions}

We identified a QTL associated with colonization 1 day post-infection by $\mathrm{O} 157: \mathrm{H} 7$ on murine $\mathrm{Chr} 9$. The identification of this QTL suggests that host genetics affect STEC O157:H7 colonization levels in mice

\section{Additional files}

Additional file 1: Figure S1. Colonization levels of 14 BXD strains tested from JAX and UC. The individual colonization levels of mice from the 14 BXD strains that were tested from both JAX and UC are depicted. Mice from JAX are shown as red squares and mice from UC are shown as black circles. The colonization levels from both sources overlap, which supports the finding that there was no difference in colonization level depending on the source of the mice, Additional file 2: Table S1. (TIFF $320 \mathrm{~kb}$ )

Additional file 2: Table S1. Analysis of variance for mean colonization day 1. (XLSX $40 \mathrm{~kb})$

Additional file 3: Figure S2. Heat map of all mapped traits across the murine genome. The QTL heat map for all members of the cluster tree, from mouse Chr 1 to distal $\mathrm{Chr} \mathrm{X}$. The more intense colors mark chromosomal regions with comparatively high linkage statistics and the spectrum encodes the allelic effect. Each individually colored line in the vertical column indicates the genome-wide $p$ value computed on the basis of 5000 permutations (significant $p$ values are indicated by colors at the right end of the spectrum). (TIFF $258 \mathrm{~kb}$ )

\section{Competing interests}

The authors declare that they have no competing interests.

\section{Authors' contributions}

Conceived and designed the experiments: LMR, NFA, ADO, MK, ARM-C. Did the experiments: LMR. Analyzed the data: LMR, NFA, ADO, ARM-C. Wrote the paper: LMR, NFA, ADO, ARM-C. All authors read and approved the final manuscript.

\section{Acknowledgements}

We thank Stephen Darnell for assistance with animal work.

The opinions or assertions contained herein are the private ones of the authors and are not to be construed as official or reflecting the views of the Department of Defense, the Uniformed Services University of the Health Sciences, or the National Institutes of Health.

This work was supported by Uniformed Services University Intramural Grant R073RV to ADO, National Institutes of Health grant R37 Al020148 to ADO, and University of Cincinnati College of Medicine \& The Cincinnati VA Medical Center Merit Award to MK.

\section{Author details}

'Department of Microbiology and Immunology, Uniformed Services University of the Health Sciences, Bethesda, MD, USA. ${ }^{2}$ University of Cincinnati College of Medicine \& Cincinnati VA Medical Center, Cincinnati, $\mathrm{OH}$, USA. ${ }^{3}$ Department Microbiology and Immunology, Faculty of Pharmacy, Cairo University, Cairo, Egypt. ${ }^{4}$ Department of Basic Biomedical Sciences, University of North Dakota, Grand Forks, ND, USA.

Received: 14 July 2015 Accepted: 22 October 2015

Published online: 16 November 2015

\section{References}

1. Scallan E, Hoekstra RM, Angulo FJ, Tauxe RV, Widdowson MA, Roy SL, et al. Foodborne illness acquired in the United States-major pathogens. Emerg Infect Dis. 2011;17(1):7-15.
2. Endo $Y$, Tsurugi $K$, Yutsudo T, Takeda Y, Ogasawara T, Igarashi K. Site of action of a Vero toxin (VT2) from Escherichia coli O157:H7 and of Shiga toxin on eukaryotic ribosomes. RNA N-glycosidase activity of the toxins. Eur J Biochem. 1988;171(1-2):45-50.

3. Nataro JP, Kaper JB. Diarrheagenic Escherichia coli. Clin Microbiol Rev. 1998;11(1):142-201.

4. Judge NA, Mason HS, O'Brien AD. Plant cell-based intimin vaccine given orally to mice primed with intimin reduces time of Escherichia coli 0157:H7 shedding in feces. Infect Immun. 2004;72(1):168-75.

5. McKee ML, Melton-Celsa AR, Moxley RA, Francis DH, O'Brien AD. Enterohemorrhagic Escherichia coli O157:H7 requires intimin to colonize the gnotobiotic pig intestine and to adhere to HEp-2 cells. Infect Immun. 1995;63(9):3739-44.

6. Cornick NA, Booher SL, Moon HW. Intimin facilitates colonization by Escherichia coli O157:H7 in adult ruminants. Infect Immun. 2002;70(5):2704-7.

7. Dean-Nystrom EA, Bosworth BT, Moon HW, O'Brien AD. Escherichia coli 0157:H7 requires intimin for enteropathogenicity in calves. Infect Immun. 1998;66(9):4560-3.

8. Aslam M, Nattress F, Greer G, Yost C, Gill C, McMullen L. Origin of contamination and genetic diversity of Escherichia coli in beef cattle. Appl Environ Microbiol. 2003;69(5):2794-9.

9. Armstrong GL, Hollingsworth J, Morris Jr JG. Emerging foodborne pathogens: Escherichia coli O157:H7 as a model of entry of a new pathogen into the food supply of the developed world. EpidemiolRev. 1996;18(1):29-51.

10. Hancock DD, Besser TE, Rice DH, Tarr PI. Ecology of Escherichia coli O157:H7 in cattle and impact of management practices. In: Kaper JB, O'Brien AD, editors. Escherichia coli 0157:H7 and other Shiga toxin-producing E coli strains. Washington, D.C: American Society for Microbiology; 1998. p. 85-91.

11. Barlow RS, Gobius KS, Desmarchelier PM. Shiga toxin-producing Escherichia coli in ground beef and lamb cuts: results of a one-year study. Int J Food Microbiol. 2006;111(1):1-5.

12. Doyle MP, Schoeni JL. Isolation of Escherichia coli O157:H7 from retail fresh meats and poultry. Appl Environ Microbiol. 1987;53(10):2394-6.

13. Michino H, Araki K, Minami S, Takaya S, Sakai N, Miyazaki M, et al. Massive outbreak of Escherichia coli 0157:H7 infection in schoolchildren in Sakai City, Japan, associated with consumption of white radish sprouts. Am J Epidemiol. 1999;150(8):787-96.

14. Cody SH, Glynn MK, Farrar JA, Cairns KL, Griffin PM, Kobayashi J, et al. An outbreak of Escherichia coli 0157:H7 infection from unpasteurized commercial apple juice. Ann Intern Med. 1999;130(3):202-9.

15. Friesema I, Sigmundsdottir G, van der Zwaluw K, Heuvelink A, Schimmer B, de Jager $C$, et al. An international outbreak of Shiga toxin-producing Escherichia coli 0157 infection due to lettuce, September-October 2007. Euro Surveill. 2008;13:50,

16. King LA, Nogareda F, Weill FX, Mariani-Kurkdjian P, Loukiadis E, Gault G, et al. Outbreak of Shiga toxin-producing Escherichia coli O104:H4 associated with organic fenugreek sprouts, France, june 2011. Clin Infect Dis. 2012;54(11):1588-94. doi:10.1093/cid/cis255.

17. Breuer T, Benkel DH, Shapiro RL, Hall WN, Winnett MM, Linn MJ, et al. A multistate outbreak of Escherichia coli 0157:H7 infections linked to alfalfa sprouts grown from contaminated seeds. Emerg Infect Dis. 2001;7(6):977-82.

18. Tarr PI, Gordon CA, Chandler WL. Shiga-toxin-producing Escherichia coli and haemolytic uraemic syndrome. Lancet. 2005;365(9464):1073-86.

19. Gould LH, Demma L, Jones TF, Hurd S, Vugia DJ, Smith K, et al. Hemolytic uremic syndrome and death in persons with Escherichia coli O157:H7 infection, foodborne diseases active surveillance network sites, 2000-2006. Clin Infect Dis. 2009:49(10):1480-5.

20. Bell BP, Griffin PM, Lozano P, Christie DL, Kobayashi JM, Tarr PI. Predictors of hemolytic uremic syndrome in children during a large outbreak of Escherichia coli O157:H7 infections. Pediatrics. 1997;100(1), E12.

21. Karmali MA. Infection by verocytotoxin-producing Escherichia coli. Clin Microbiol Rev. 1989;2(1):15-38.

22. Paton JC, Paton AW. Pathogenesis and diagnosis of Shiga toxin-producing Escherichia coli infections. Clin Microbiol Rev. 1998;11(3):450-79.

23. Churchill GA, Airey DC, Allayee H, Angel JM, Attie AD, Beatty J, et al. The collaborative cross, a community resource for the genetic analysis of complex traits. Nat Genet. 2004;36(11):1133-7.

24. Peirce JL, Lu L, Gu J, Silver LM, Williams RW. A new set of BXD recombinant inbred lines from advanced intercross populations in mice. BMC Genet. 2004;5:7. 
25. Churchill GA, Doerge RW. Empirical threshold values for quantitative trait mapping. Genetics. 1994;138(3):963-71.

26. Shifman S, Bell JT, Copley RR, Taylor MS, Williams RW, Mott R, et al. A high-resolution single nucleotide polymorphism genetic map of the mouse genome. PLoS Bio. 2006;4(12), e395.

27. Waterston RH, Lindblad-Toh K, Birney E, Rogers J, Abril JF, Agarwal P, et al. Initial sequencing and comparative analysis of the mouse genome. Nature. 2002:420(6915):520-62

28. Wang X, Agarwala R, Capra JA, Chen Z, Church DM, Ciobanu DC, et al. High-throughput sequencing of the DBA/2J mouse genome. BMC Bioinformatics. 2010;11(4):07.

29. Celera. Celera completes assembly of mouse genome. Rockville: CELERA; 2001.

30. Wu Y, Williams EG, Dubuis S, Mottis A, Jovaisaite V, Houten SM, et al. Multilayered genetic and omics dissection of mitochondrial activity in a mouse reference population. Cell. 2014;158(6):1415-30.

31. Lu L, Wei L, Peirce JL, Wang X, Zhou J, Homayouni R, et al. Using gene expression databases for classical trait QTL candidate gene discovery in the BXD recombinant inbred genetic reference population: mouse forebrain weight. BMC Genom. 2008;9:444.

32. Klein RF. Genetic regulation of bone mineral density in mice. J Musculoskelet Neuronal Interact. 2002;2(3):232-6.

33. Crabbe JC, Belknap JK, Buck KJ, Metten P. Use of recombinant inbred strains for studying genetic determinants of responses to alcohol. Alcohol Alcohol. 1994;2:67-71.

34. Wang LS, Jiao Y, Huang Y, Liu XY, Gibson G, Bennett B, et al. Critical evaluation of transcription factor Atf2 as a candidate modulator of alcohol preference in mouse and human populations. GMR. 2013;12(4):5992-6005.

35. Boon AC, de Beauchamp J, Hollmann A, Luke J, Kotb M, Rowe S, et al. Host genetic variation affects resistance to infection with a highly pathogenic H5N1 influenza A virus in mice. J Virol. 2009;83(20):10417-26.

36. Abdeltawab NF, Aziz RK, Kansal R, Rowe SL, Su Y, Gardner L, et al. An unbiased systems genetics approach to mapping genetic loci modulating susceptibility to severe streptococcal sepsis. PLoS Pathog. 2008;4(4), e1000042.

37. Aziz RK, Kansal R, Abdeltawab NF, Rowe SL, Su Y, Carrigan D, et al. Susceptibility to severe Streptococcal sepsis: use of a large set of isogenic mouse lines to study genetic and environmental factors. Genes Immun. 2007:8(5):404-15.

38. Zumbrun EE, Abdeltawab NF, Bloomfield HA, Chance TB, Nichols DK, Harrison $\mathrm{PE}$, et al. Development of a murine model for aerosolized ebolavirus infection using a panel of recombinant inbred mice. Viruses 2012;4(12):3468-93.

39. Mural RJ, Adams MD, Myers EW, Smith HO, Miklos GL, Wides R, et al. A comparison of whole-genome shotgun-derived mouse chromosome 16 and the human genome. Science. 2002;296(5573):1661-71.

40. Rosenthal N, Brown S. The mouse ascending: perspectives for humandisease models. Nat Cell Biol. 2007;9(9):993-9.

41. Sieberts SK, Schadt EE. Moving toward a system genetics view of disease. Mamm Genome : Off J Int Mamm Genome Soc. 2007;18(6-7):389-401.

42. U.S. National Research Council CftUotGftCaUoLA, Institute for Laboratory Animal Research. Guide for the care and use of laboratory animals. 8th ed. Washington, DC: National Academies Press; 2011.

43. Griffin PM, Ostroff SM, Tauxe RV, Greene KD, Wells JG, Lewis JH, et al. IInesses associated with Escherichia coli O157:H7 infections. A broad clinical spectrum. Ann Intern Med. 1988:109(9):705-12

44. Gunzer F, Bohn U, Fuchs S, Muhldorfer I, Hacker J, Tzipori S, et al. Construction and characterization of an isogenic slt-ii deletion mutant of enterohemorrhagic Escherichia coli. Infect Immun. 1998;66(5):2337-41.

45. Robinson CM, Sinclair JF, Smith MJ, O'Brien AD. Shiga toxin of enterohemorrhagic Escherichia coli type 0157:H7 promotes intestinal colonization. Proc Natl Acad Sci U S A. 2006;103(25):9667-72.

46. Mohawk KL, Melton-Celsa AR, Zangari T, Carroll EE, O'Brien AD. Pathogenesis of Escherichia coli 0157:H7 strain 86-24 following oral infection of BALB/C mice with an intact commensal flora. Microb Pathog. 2010;48:131-42.

47. Chen H, Sharp BM. Content-rich biological network constructed by mining PubMed abstracts. BMC Bioinformatics. 2004;5:147.

48. Moore KJ, Nagle DL. Complex trait analysis in the mouse: The strengths, the limitations and the promise yet to come. Annu Rev Genet. 2000;34:653-86.

49. Maglott D, Ostell J, Pruitt KD, Tatusova T. Entrez Gene: gene-centered information at NCBI. Nucleic Acids Res. 2005:33(Database issue):D54-8.
50. Hennings SJ, Hird FJ. Concentrations and metabolism of volatile fatty acids in the fermentative organs of two species of kangaroo and the guinea-pig. Br J Nutr. 1970;24(1):145-55.

51. Firmansyah A, Penn D, Lebenthal E. Isolated colonocyte metabolism of glucose, glutamine, n-butyrate, and beta-hydroxybutyrate in malnutrition. Gastroenterology. 1989:97(3):622-9.

52. Scheppach W. Effects of short chain fatty acids on gut morphology and function. Gut. 1994;35(1 Suppl):S35-8.

53. De Preter V, Arijs I, Windey K, Vanhove W, Vermeire S, Schuit F, et al. Impaired butyrate oxidation in ulcerative colitis is due to decreased butyrate uptake and a defect in the oxidation pathway. Inflamm Bowel Dis. 2012;18(6):1127-36.

54. Nakanishi N, Tashiro K, Kuhara S, Hayashi T, Sugimoto N, Tobe T. Regulation of virulence by butyrate sensing in enterohaemorrhagic Escherichia coli. Microbiology. 2009;155(Pt 2):521-30

55. Zumbrun SD, Melton-Celsa AR, Smith MA, Gilbreath JJ, Merrell DS, O'Brien AD. Dietary choice affects Shiga toxin-producing Escherichia coli (STEC) O157:H7 colonization and disease. Proc Natl Acad Sci U S A. 2013;110(23):E2126-33.

56. Helbing T, Rothweiler R, Heinke J, Goetz L, Diehl P, Zirlik A, et al. BMPER is upregulated by statins and modulates endothelial inflammation by intercellular adhesion molecule-1. Arterioscler Thromb Vasc Biol. 2010;30(3):554-60.

57. Pi X, Lockyer P, Dyer LA, Schisler JC, Russell B, Carey S, et al. Bmper inhibits endothelial expression of inflammatory adhesion molecules and protects against atherosclerosis. Arterioscler Thromb Vasc Biol. 2012;32(9):2214-22.

58. Helbing T, Rothweiler R, Ketterer E, Goetz L, Heinke J, Grundmann S, et al. BMP activity controlled by BMPER regulates the proinflammatory phenotype of endothelium. Blood. 2011;118(18):5040-9.

59. Blease K, Burke-Gaffney A, Hellewell PG. Modulation of cell adhesion molecule expression and function on human lung microvascular endothelial cells by inhibition of phosphodiesterases 3 and $4 . \mathrm{Br} \mathrm{J}$ Pharmacol. 1998;124(1):229-37.

60. Sanz MJ, Cortijo J, Morcillo EJ. PDE4 inhibitors as new anti-inflammatory drugs: effects on cell trafficking and cell adhesion molecules expression. Pharmacol Ther. 2005;106(3):269-97.

61. Okayama A, Mikasa K, Matsui N, Higashi N, Miyamoto M, Kita E. An interventional approach to block brain damage caused by Shiga toxin-producing Escherichia coli infection, by use of a combination of phosphodiesterase inhibitors. I Infect Dis. 2004;190(12):2129-36.

62. Velasquez S, Eugenin EA. Role of Pannexin-1 hemichannels and purinergic receptors in the pathogenesis of human diseases. Front Physiol. 2014;5:96.

63. Ohbuchi T, Hohchi N, Ohkubo J, Hashida K, Koizumi H, Wakasugi T, et al. Identification of pannexins in rat nasal mucosa. Allergy Rhinology. 2013:4(2):e63-5.

64. Ghosh SK, McCormick TS, Eapen BL, Yohannes E, Chance MR, Weinberg A. Comparison of epigenetic profiles of human oral epithelial cells from HIV-positive (on HAART) and HIV-negative subjects. Epigenetics : Off J DNA Methylation Soc. 2013;8(7):703-9.

65. Tolg C, Sabha N, Cortese R, Panchal T, Ahsan A, Soliman A, et al. Uropathogenic $E$. coli infection provokes epigenetic downregulation of CDKN2A (p16INK4A) in uroepithelial cells. Lab Investig: J Tech Methods Pathol. 2011;91(6):825-36.

\section{Submit your next manuscript to BioMed Central and take full advantage of:}

- Convenient online submission

- Thorough peer review

- No space constraints or color figure charges

- Immediate publication on acceptance

- Inclusion in PubMed, CAS, Scopus and Google Scholar

- Research which is freely available for redistribution 